\title{
Das Demenzrisiko nimmt zu
}

Fragestellung: Ist Vorhofflimmern ein Risikofaktor für die Entwicklung einer Demenz?

Hintergrund: Vorhofflimmern ist ein wichtiger Risikofaktor für ischämische Insulte. Ischämische Insulte wiederum sind ein wesentlicher Prädiktor für die Entwicklung einer Demenz und zwar sowohl einer degenerativen wie einer vaskulären Demenz. Es gibt eine Reihe von Registern und Studien, die zeigen, dass Patienten mit Vorhofflimmern ein höheres Risiko haben, später eine Demenz zu entwickeln. Es gibt allerdings bisher wenige populationsbezogene Studien, die diesen Zusammenhang untersucht haben und für das Vorhandensein oder die Abwesenheit eines ischämischen Insults korrigieren.

Patienten und Methodik: Die Publikation stammt aus der Rotterdam-Studie. Dies ist eine populationsbezogene Studie in Rotterdam, die chronische Krankheiten bei älteren Menschen prospektiv untersucht. Die Studie begann 1989 und schloss 7.983 Menschen im Alter über 55 Jahre ein, die zustimmten, alle drei Jahre nachuntersucht zu werden. Bei allen Probanden wurde jeweils zu Beginn und bei den nachfolgenden Untersuchungen ein EKG durchgeführt. Wenn Vorhofflimmern registriert wurde, wurde dieses vermerkt. Bei allen Patienten erfolgte bei Einschluss in die Studie eine Mi-

De Bruijn RF, Heeringa J, Wolters FJ et al. Association between atrial fibrillation and dementia in the general population. JAMA Neurol 2015; doi: 10.1001/jamaneurol.2015.2161. (abstract) ni-Mental State Examination (MMSE) als Screening-Methode. Bei allen Patienten mit einem MMSE-Wert $<26$ wurde dann als ausführlichere Untersuchung der Cambridge Examination for Mental Disorders of the Elderly-
Test durchgeführt. Dieser Test wurde dann 2014 wiederholt. Ausgeschlossen wurden Probanden, bei denen kein DemenzScreening bei Einschluss erfolgt war sowie Probanden, bei denen bereits eine Demenz bekannt war.

Ergebnisse: Bei 490 Patienten waren keine Informationen vorhanden, ob ein Vorhofflimmern bestand. Die Studie beruhte daher auf den Daten von 6.514 Patienten, die bei Einschluss in die Studie keine Demenz aufwiesen. Für die Analyse wurde für Alter, Geschlecht, kardiovaskuläre Risikofaktoren oder Schlaganfälle adjustiert. Der primäre Studienendpunkt war eine neu aufgetretene Demenz. Bei Studieneinschluss bestand bei 4,9\% der Teilnehmer Vorhofflimmern. Während der Beobachtungszeit entwickelten insgesamt 994 Teilnehmer (15,3\%) eine Demenz.

Vorhofflimmern erhöhte das Risiko einer Demenzentwicklung um den Faktor 1,33. Von den Studienteilnehmern, die bei Studieneinschluss kein Vorhofflimmern aufwiesen, entwickelten $11,7 \%$ im weiteren Verlauf Vorhofflimmern und bei $15 \%$ $(\mathrm{n}=932)$ trat eine Demenz auf. Hier betrug die Risikoerhöhung 1,18 für Patienten unter 67 Jahren und 1,12 (nicht signifikant) für diejenigen älter als 67 Jahre.

Das Risiko eine Demenz zu entwickeln, war mit der Dauer des Vorhofflimmerns assoziiert. Allerdings galt dies ausschließlich für Patienten unter 67 Jahren, aber nicht für Patienten über 67 Jahre.

Schlussfolgerungen: In der populationsbezogenen RotterdamStudie fand sich eine Assoziation zwischen Vorhofflimmern und der Entwicklung einer Demenz, die ganz überwiegend bei den Patienten im Alter unter 67 Jahren beobachtet wurde.

\section{- Kommentar von Hans-Christoph Diener, Essen}

\section{Ursache der Demenz bei Vorhofflimmern sind oft Schlaganfälle}

Diese Publikation hat viel Aufregung in der kardiologischen Community hervorgerufen. Dabei wurde übersehen, dass der Zusammenhang zwischen Vorhofflimmern und Demenz seit langem bekannt ist. Bei einem Teil der Menschen, die eine Demenz entwickeln, liegt die Ursache darin, dass sie aufgrund von Vorhofflimmern Schlaganfälle erlitten haben.

Bei Menschen mit Vorhofflimmern sind interessanterweise auch Microbleeds, lakunäre Infarkte und White matter lesions häufiger als bei Menschen ohne Vorhofflimmern, wobei dies auf gleichzeitig bestehende vaskuläre Risikofaktoren zurückzuführen ist. Leider konnten die Autoren aus Rotterdam nicht unterscheiden, ob es sich bei den Patienten, die eine Demenz entwickelten, um eine degenerative Alzheimer-Demenz oder um eine vaskuläre Demenz handelte.

Völlig unbekannt ist, ob die optimale Antikoagulation bei Patienten mit Vorhofflimmern die zukünftige Entwicklung einer Demenz verhindern kann. Randomisierte klinische Studien zu dieser Fragestellung sind kaum zu erwarten, da hier die Beobachtungszeit zwischen 10 und 15 Jahren liegen müsste.

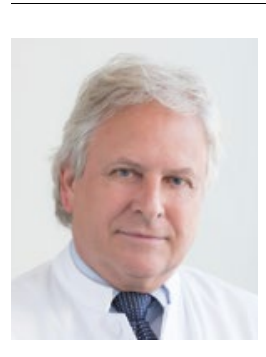

Prof. Dr. med. Hans-Christoph

Diener, Essen

Direktor der Klinik für Neurologie,

Universitätsklinikum Essen

E-Mail:h.diener@uni-essen.de 